\title{
OA10.02. Yoga for women with breast cancer undergoing radiotherapy (XRT): a randomized clinical trial with an active stretching control group
}

\author{
L Cohen ${ }^{1 *}$, K Chandwani ${ }^{2}$, N Raghuram ${ }^{3}$, R Haddad ${ }^{1}$, G Perkins ${ }^{1}$, A Spelman ${ }^{1}$, R Nagarathna ${ }^{4}$, K Johnson ${ }^{1}$, \\ A Fortier ${ }^{1}$, B Arun ${ }^{1}$, Q Wei ${ }^{1}$, C Kirschbaum ${ }^{5}$, H Nagendra $^{4}$
}

From International Research Congress on Integrative Medicine and Health 2012

Portland, Oregon, USA. 15-18 May 2012

\section{Purpose}

We examined the effects of yoga on buffering changes in QOL and cortisol slope in women with breast cancer undergoing (XRT).

\section{Methods}

Patients with stage 0-III disease were recruited prior to XRT (baseline) and randomized to one of three groups: Yoga $(Y G-n=53)$ or Stretching program $($ STR-n=56) 3 times a week for 6 weeks during XRT or Waitlist Control (WLC-n=54). Self-report measures of fatigue (BFI), depression (CESD), QOL (SF-36), benefit finding (BF), and spirituality (FACT-SP) were completed and saliva collected at baseline, end of treatment, and 1, 3, and 6 months later. We examined change from baseline for questionnaires and slope analyses for cortisol.

\section{Results}

By the end of XRT, the YG and STR groups had a reduction in fatigue while the WLC had an increase (YG: -0.23, STR: -0.45, WLC: 0.52; p's<.05). At 1, 3, and 6 months after XRT, the YG group had greater increases in SF-36 physical functioning compared to both STR and WLC (1 month: 5.8, 2.0, 0.8; 3 months: 6.5, 3.4, -0.2; 6 months: $6.1,3.4,1.1$; p's<.05). The outcomes were similar for SF-36 general health scores. By 3 and 6 months after XRT, there were significant increases in BF for the YG group (3 months: $3.0,-2.6,-2.5 ; 6$ months: $1.1 ;-3.9 ;-4.7 ; \mathrm{p}$ 's $<.05)$. There were no differences for spirituality and depression. Cortisol slope was steepest for the YG group compared to the STR and WLC groups (end of XRT: $-0.10,-0.08,-0.08 ; 1$ month: -0.10 , $-0.09,-0.06 ; \mathrm{p}$ 's $<.01)$.

\section{Conclusion}

Yoga buffered changes associated with XRT in terms of fatigue, QOL and benefit finding, and resulted in steeper cortisol slopes, while stretching resulted in only modest benefits. This is the first yoga study in oncology to include an active control group, suggesting that the benefits of yoga are due to more than simple stretching or other indirect effects.

\section{Author details \\ ${ }^{1}$ The University of Texas MD Anderson Cancer Center, Houston, USA. ${ }^{2}$ Swami Vivekananda Yoga Research Foundation, Bangalore, India. ${ }^{3}$ Vivekananda Yoga University, Bangalore, India. "Vivekananda Yoga Research Foundation, Bangalore, India. ${ }^{5}$ Technical University of Dresden, Dresden, Germany.}

Published: 12 June 2012

doi:10.1186/1472-6882-12-S1-038

Cite this article as: Cohen et al:: OA10.02. Yoga for women with breast cancer undergoing radiotherapy (XRT): a randomized clinical trial with an active stretching control group. BMC Complementary and Alternative Medicine 2012 12(Suppl 1):O38.

'The University of Texas MD Anderson Cancer Center, Houston, USA

Full list of author information is available at the end of the article

C 2012 Cohen et al; licensee BioMed Central Ltd. This is an Open Access article distributed under the terms of the Creative Commons 